\title{
Epidemiology and pathogenesis of clinical spectrum of Japanese encephalitis Usha Kant Misra
}

Address: Department of Neurology, Sanjay Gandhi Post Graduate Institute of Medical Sciences, Lucknow, Uttar Pradesh, 226014, India

Email: Usha Kant Misra - drukmisra@rediffmail.com; ukmisra@sgpgi.ac.in

from Infectious diseases of the nervous system: pathogenesis and worldwide impact

Paris, France. 10-13 September 2008

Published: 23 September 2008

BMC Proceedings 2008, 2(Suppl I):S30

This abstract is available from: http://www.biomedcentral.com/I753-656I/2/SI/S30

(c) 2008 Misra; licensee BioMed Central Ltd.

Japanese encephalitis (JE) is the most important endemic encephalitis in the world affecting over 50,000 patients annually in Southeast Asia. One-third of JE patients die and half of the survivors have neurological sequelae. Japanese Encephalitis spreads by mosquito bite and is maintained by wild and domestic birds and pigs. Humans are dead end host as they don't spread the disease because of brief viremia. JE is generally a disease of the children but affects the adults also in the newly invaded areas. JE occurs in two distinct patterns, in the northern regions as summer epidemics and in southern regions as sporadic or endemic disease. Occurrence of JE has been linked to rainfall and high temperature when mosquito density increases. In north India, JE occurs in the months of August to October. The areas of occurrence of JE have been increasing because of pig farming, paddy cultivation, poverty and poor sanitation. JE has been controlled in Japan, Korea and Taiwan because of vaccination, improvement in socioeconomic status and improved agricultural practice and pig farming. In North India, JE vaccination program has been initiated now.

The clinical picture of JE begins with a non-specific prodrome. There are 25-1000 cases of subclinical infection for each JE patient. Cranial MRI reveals high frequency of thalamic (94\%), basal ganglia (35\%) and brainstem (59\%) involvement in admitted patients and thalamic involvement is highly suggestive of JE in endemic areas in post monsoon period. Seizure occurs in $40 \%$ JE patients, more commonly in children which may be generalized tonic clonic or partial. In Vietnamese children with JE presence of seizure was associated with poor outcome. After 1-4 week of onset, about 30\% JE patients develop a variety of movement disorders such as transient form of Parkinsonism and dystonia more commonly and chorea, athetosis and myoclonus rarely. CSF norepinephrine, dopamine, and HVA levels are significantly reduced in JE patients with movement disorders compared to controls but there is no difference in CSF catacholamine levels in patients with parkinsonian features and dystonia. A number of JE patients develop anterior horn cell involvement simulating poliomyelitis. Depth of coma and anterior horn cell involvement are associated with poor outcome. Neurophysiological investigations such as electoencephalography, evoked potentials, nerve conduction studies, EMG and SPECT studies have helped in understanding the pathophysiology of JE better and shall be discussed. 